\title{
Magnetic Levitation System with Interface for Position Control
}

\author{
Nino Vega, MSc ${ }^{1}$, Pablo Parra, Ph.D2 ${ }^{2}$, Teddy Negrete, MSc ${ }^{3}$, Pablo Fuentes, Ing ${ }^{4}$, Josep Peñafiel, Ing 5 . \\ ${ }^{1}$ Universidad Católica de Santiago de Guayaquil, Ecuador, nino.vega@ucsg.edu.ec, teddynegrete@gmail.com \\ josephpenafiel@outlook.com, \\ ${ }^{2}$ Universidad Católica de Santiago de Guayaquil, Ecuador, pablitoparrarosero@gmail.com, f.pablo1@hotmail.com,
}

\begin{abstract}
This paper exposes the development of a magnetic levitation plant and interface for control the position of a suspended permanent magnet and for monitor the control signal. Its interface have a didactic purpose to allow the students to observe how the control variable of the system changes with perturbations, as well as the design of a PD controller for a linear approximation of a non-linear system. The implementation is composed of the controller circuit (OPAMP and Hall Effect sensor), the power circuit (transistor and electromagnet), and a microcontroller to communicate with the graphical interface through computer. The graphical interface was developed with the GUI editor of MATLAB. Different tests have been performed with the prototype, and they verified that the designed controller worked properly keeping the levitating object in the desired position.
\end{abstract}

Keywords-- Nonlinear system, Magnetic Levitation, controller design, MATLAB GUI.

\section{INTRODUCCIÓN}

Un sistema de levitación magnética es uno de los primeros sistemas que se estudia cuando se quiere abarcar el campo de control de sistemas no lineales, debido a su sencillez respecto a otros del mismo grupo. La inestabilidad inherente de este sistema constituye un reto, en especial cuando de estudiantes se trata, sin duda esto los motiva a continuar con su proceso de aprendizaje y a entender la necesidad e importancia del control en los sistemas reales.

La levitación magnética ha causado un gran impacto en diversas aplicaciones tales como: en la ingeniería de transporte, con la creación de trenes que levitan magnéticamente y que pueden transportarse a velocidades mucho más altas que los trenes tradicionales, en la ingeniería ambiental, aeroespacial, militar, nuclear, civil, química, eléctrica, [1].

El principio básico de operación de un sistema de levitación es aplicar un voltaje a un electroimán para mantener un objeto ferromagnético levitando [2], para llegar a la levitación de este objeto, se requiere de un controlador en tiempo-real el cual debe ser capaz de mantener en equilibrio las dos fuerzas principales que actúan sobre el objeto a levitar, la fuerza gravitatoria y la fuerza electromagnética; sólo en el momento que se han cancelado el objeto estará suspendido en el aire [3] [4].

Para lograr desarrollar un proyecto de levitación magnética es necesario obtener el modelo matemático que describe la dinámica de la planta [5] [6]. y alrededor de él, diseñar un controlador, para compensar todas las inestabilidades que se observan mediante la evaluación del modelo matemático conseguido. La implementación se la realiza en en dos partes fundamentales: modelado del sistema y diseño del controlador. [7].

Comercialmente, existen diversas plantas de este sistema a la venta, que tienen fines educativos y de investigación, por ejemplo, la planta desarrollada por Feedback Instruments 2009 [8]. Cuyo valor unitario está sobre los $\$ 6000$ [9]., que limita el poder adquirirla; sin embargo, esta planta cuenta con varias características, como las interfaces de control, que tienen comunicación a diferentes aplicaciones como MATLAB o LabView que aumentan su costo. Otra planta con fines educativos que se puede encontrar, es la desarrollada por Zeltom [10] [11], la cual con un precio de aproximadamente $\$ 40$, es la más asequible económicamente; sin embargo, ésta carece de una interfaz de control gráfica, ni algún medio para realizar el seguimiento de la señal de control.

En este artículo, se pretende mostrar el desarrollo un prototipo de bajo costo con ayuda de los componentes que utiliza la planta desarrollada por Zeltom [11], y que incorpora características importantes para el análisis de este sistema, tales como: la monitorización, seguimiento de las variables del proceso y modificación en tiempo real de la referencia.

\section{DESCRIPCIÓN DEL EQUIPO}

El sistema de Levitación Magnética se desarrolló en cuatro etapas: modelado matemático del sistema, diseño del controlador, diseño del prototipo, y desarrollo de la interfaz gráfica.

\section{A. Modelado Matemático del Sistema.}

En la Figura 1 se muestra los elementos más importantes de una planta de levitación magnética, las ecuaciones que intervienen en el desarrollo del modelo matemático son las ecuaciones 1, 2, 3 y 4 se tomaron como base las ecuaciones dadas por el modelo de [12] [13] [114] [15.]En la ecuación 1 se aplicación la Ley de voltaje de Kirchhoff en el circuito equivalente del electroimán, la ecuación 2 es la aplicación de la segunda Ley de Newton que corresponden a las fuerzas que actúan sobre el sistema, y la ecuación 3 es aquella que define el comportamiento del sensor de efecto Hall en el sistema [10] [16]. Las variable $v i, R$ y $L$ es el voltaje, corriente, resistencia y la inductancia del electroimán, respectivamente, $m$ es la masa del objeto a levitar, $x$ es la altura a la que se encuentra el objeto, $g$ es la gravedad, $F_{m}$ es la fuerza electromagnética, $\boldsymbol{e}_{x}$ es el voltaje de salida del sensor $\alpha, \beta$ y $\gamma$ son constantes del 
sensor de efecto Hall, $n$ es el ruido en el proceso que altera las mediciones, y $K_{m}$ es una constante del sistema.

$$
\begin{aligned}
& \nu=R i+\frac{L d i}{d t} \\
& m \bar{x}=m g-F_{m} \\
& e=\alpha+\frac{\beta}{x^{2}}+\gamma i+n \\
& F_{m}=\frac{K_{m} i}{x^{4}}
\end{aligned}
$$

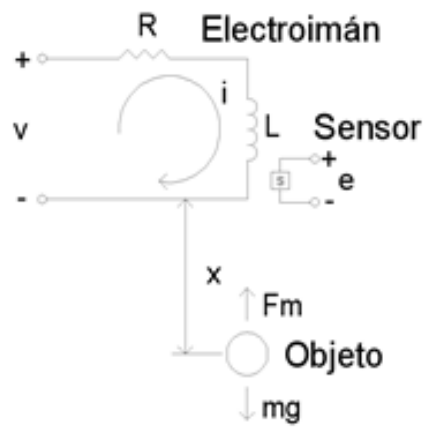

Fig. 1: Variables y fuerza que intervienen en el sistema

Las ecuaciones 3 y 4 presentan una naturaleza no lineal, al realizar la linealización de estas ecuaciones, a través de series de Taylor, alrededor de un punto de operación $\left(x_{0}, i_{0}\right)$, el resultado genera las ecuaciones 5 y 6

$$
F_{m}=\frac{K_{m} i_{0}}{x_{0}^{4}}+\frac{K_{m}}{x_{0}^{4}} \Delta i-\frac{4 K_{m} i_{0}}{x_{0}^{5}} \Delta x
$$

$\Delta e=-\frac{2 \beta}{x_{0}^{\mathrm{a}}} \Delta x+\gamma \Delta i$

Para determinar la constante $K_{m}$ es necesario suponer el equilibrio de la ecuación 2 en el punto $\left(x_{0}, i_{0}\right)$ con lo que se obtienen la ecuación 7 y 8

La función de transferencia que representa al sistema, se la expresa a través del voltaje de salida del sensor con el voltaje de entrada que se aplica al electroimán al relacionar las ecuaciones 1,2,3,4,5,6,7 se obtiene la ecuación 8 y 9

$0=m g-\frac{K_{m} i_{0}}{x_{0}^{4}}$

$\boldsymbol{K}_{m}=\frac{m g x_{0}^{4}}{\tilde{t}_{0}}$

$\frac{\Delta E(s)}{\Delta V(s)}=\frac{\frac{2 \beta}{x_{0}^{2}} K_{\mathrm{i}}+\gamma\left(m s^{2}-K_{x}\right)}{\left(m s^{2}-K_{x}\right)(L s+R)}$
$K_{\mathrm{i}}=\frac{K_{\mathrm{m}}}{x_{0}^{4}}$

Donde $K_{\mathrm{i}}$ y $K_{x}$ son las constantes dadas por las ecuaciones $10 \mathrm{y}$ 11

$K_{x}=\frac{4 K_{m} i_{0}}{x_{0}^{5}}$

Los parámetros constantes del Sistema se los ha consolidado en la tabla 1, con las cuales se obtiene la ecuación 12 que representa la función de transferencia definitiva.

TABLA I

Parámetros del sistema de levitación magnética

\begin{tabular}{|c|c|c}
\hline & Parámetro & Valor \\
\hline \multirow{2}{*}{ Electroimán } & $\mathrm{R}$ & $2.41 \Omega$ \\
\cline { 2 - 3 } & $\mathrm{L}$ & $15.02 \mathrm{mH}$ \\
\hline Objeto a levitar & $\mathrm{m}$ & $3.02 \times 10^{-3} \mathrm{~kg}$ \\
\hline $\begin{array}{c}\text { Sensor de efecto } \\
\text { Hall }\end{array}$ & $\beta$ & $2.92 \times 10^{-4} \mathrm{Vm}^{2}$ \\
\cline { 2 - 3 } & $\mathrm{\gamma}$ & $0.48 \mathrm{~V} / \mathrm{A}$ \\
\hline Punto de operación & $\mathrm{x}_{0}$ & $2 \mathrm{~cm}$ \\
\cline { 2 - 3 } & $\mathrm{i}_{0}$ & $0.27 \mathrm{~A}$ \\
\hline
\end{tabular}

$G(s)=\frac{\Delta E[s]}{\Delta V(s]}$

$=\frac{0.00145 s^{2}+5.166}{4.539 * 10^{-1} s^{x}+0.007278 s^{2}-0.08906 s-14.28}$

\section{B. Diseño del Controlador.}

La función de transferencia (ecuación 12) fue colocada en Matlab con la aplicación RLOCUS, en la que se observa la trayectoria de las raíces de la ecuación característica de la planta. Se concluye que el sistema es inestable por tener un polo del lado derecho del plano $\mathbf{s}$.

Se aplicar un controlador PD que consiste en incorporar un polo en lugar estratégico para que genere una trayectoria de lado izquierdo del plano s, con el objetivo de lograr que la planta sea estable. Después de realizar un ajuste del controlador, se logra colocar la trayectoria de las raíces del lado izquierdo, como se muestra en la figura 2 , trayectorias que tiene la misma forma del diseño elaborado por Chico y Rondón, [17].

La ganancia necesaria para eliminar el polo del lado derecho debe ser mayor a 2.76 , con un valor de ganancia $k_{e}=2.77$, la señal de salida del sistema se estabiliza con la dinámica mostrada en la figura 3 , en esta se observa que el tiempo en que se estabiliza la posición del objeto que levita es muy alto, es de aproximadamente 400 segundos. Para una planta de este tipo se necesita que el controlatpr reaccione lo más rápido posible para evitar que el objeto caiga por efecto de la gravedad, o sea completamente atraído por la fuerza

$16^{\text {th }}$ LACCEI International Multi-Conference for Engineering, Education, and Technology: "Innovation in Education and 
electromagnética. Después de varias pruebas, se concluyó que con un $k_{e}=47$, el sistema responde de manera rápida, aunque con pequeñas fluctuaciones, en la Fig. 4 muestra la respuesta del sistema a una entrada paso, con un controlador que tiene una constante $k_{c}=47$. El controlador está dado por la ecuación 13, las cuales se obtienen siguiendo el modelamiento realizado por Boundless [18]

$$
C(s)=47(0.1683 s+1)
$$

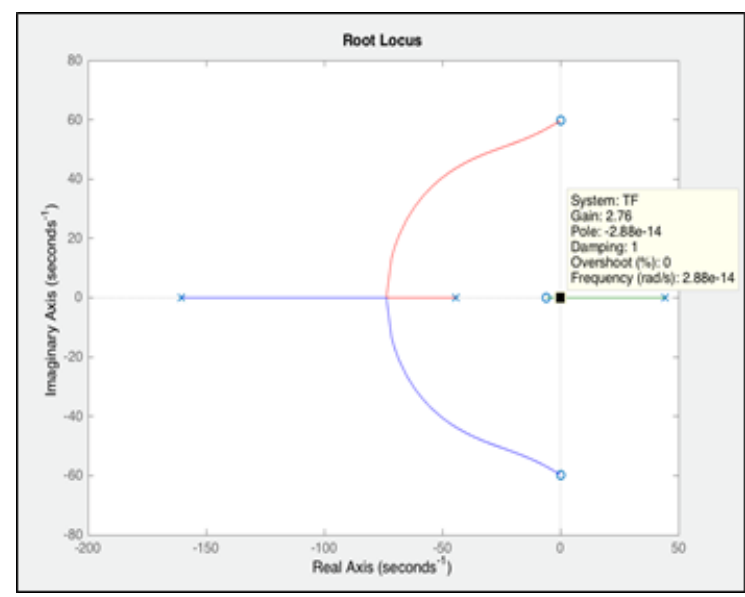

Fig.2: Trayectoria de las raíces del sistema con controlador

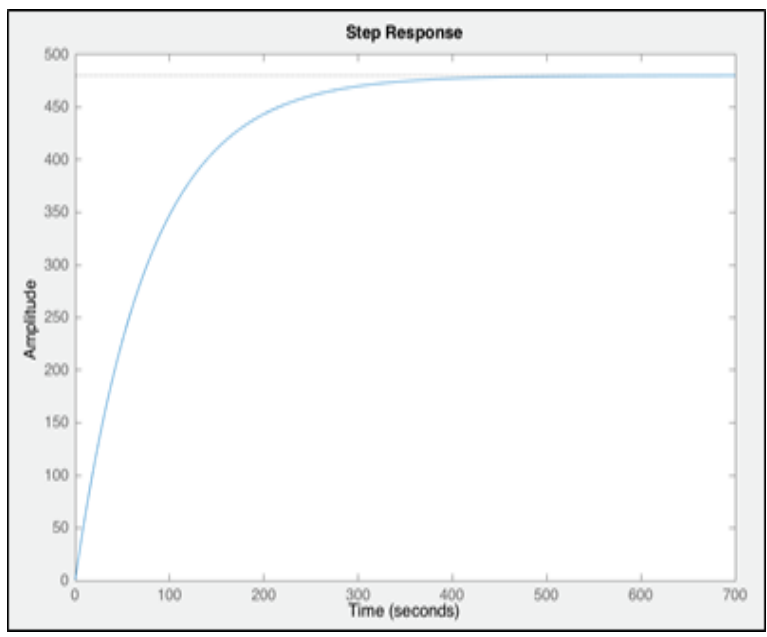

Fig. 3: Respuesta del sistema a una entrada paso

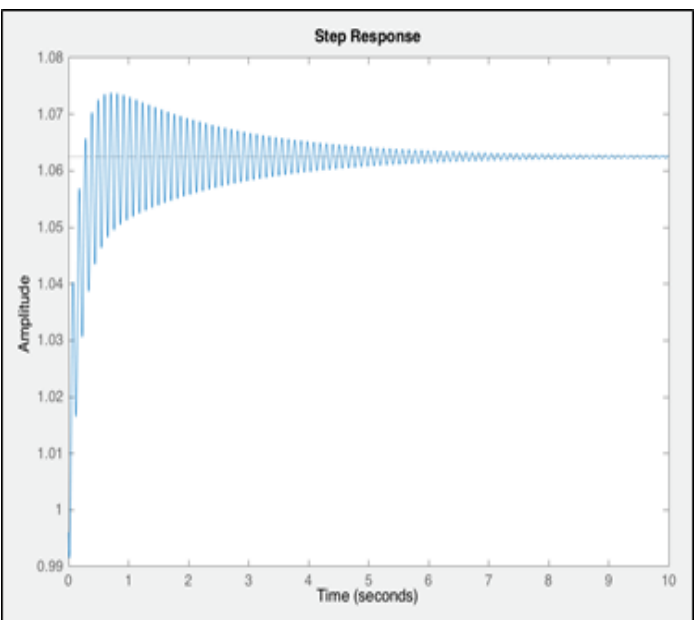

Fig. 4: Respuesta del sistema con controlador para un $\mathrm{k}_{\mathrm{c}}=47$

\section{Diseño del Prototipo}

En la figura 5 se muestra el diagrama de funcionamiento del sistema, el cual consta de, un circuito controlador que realiza la estabilización de la variable de salida de la planta de levitación, un microcontrolador placa NUCLEO-L476RG MCU [19], [20],en la que se establecer el punto de referencia y permite la comunicación con dos tipos de interfaces; una interfaz por hardware, en la que se utiliza botones, LEDs y un potenciómetro para la interacción del usuario; y una interfaz por software con la ayuda de una GUI desarrollada en MATLAB.

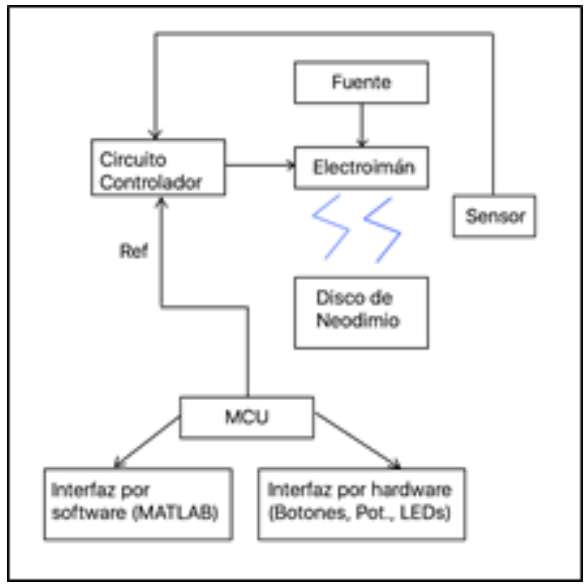

Fig. 5: Diagrama de conexiones del sistema

\section{Circuito controlador}

El circuito que controla la planta se lo ha implementado con amplificadores operacionales [21], este se divide en dos sub-circuitos. El circuito de lado derecho de la Figura 6, es un circuito diferencial que entrega como salida la señal de error del sistema entre la señal del set point y la señal que emite el sensor de la posición del objeto. Y un segundo circuito (lado derecho de la figura 6), que actúa como un controlador PD, en base a la señal de error. La señal de salida (VCOUT) es la que se aplica al circuito de fuerza del electroimán (Fig. 8) 


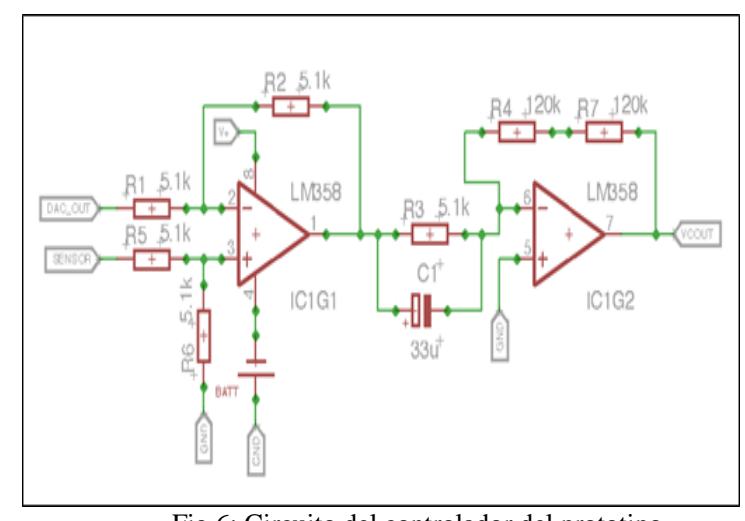

Fig.6: Circuito del controlador del prototipo

E. Implementación del Controlador Analógico

En la figura 7 se muestra el circuito que permite implementar el controlador PD de la ecuación 13, en el que se usa un amplificador operacional y una red RC

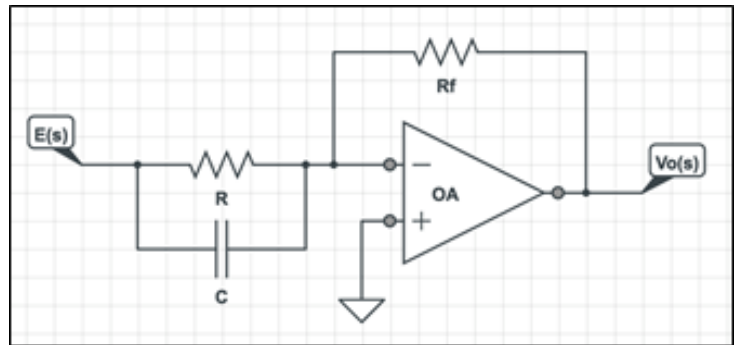

Fig.7: Estructura de un controlador PD con OPAMP

La función de transferencia de este circuito está dada por la ecuación 14 [22],

$\frac{V o(s)}{E(s)}=-\frac{R_{f}}{R}(R C s+1)$

El signo menos que se obtiene en la ecuación 14, se cancelará con el circuito previo que se coloca para obtener la señal de error (Fig 6). Se calibra los valores de resistencia y capacitancia para que la ecuación 13 y 14 sean iguales los valores obtenidos son $R=5.1 \mathrm{k} \Omega$ y $C=33 \mu F$ el proceso se muestra en la ecuación 15

$\frac{V o(s)}{E(s)}=-k_{c}(0.1638 s+1)$

Como el valor de $R=5.1 \mathrm{k} \Omega$ y que $k_{c}=R_{f} / R$, el valor de $R_{f}$ que hace que $k_{c}=47$ es el mostrado en la ecuación 16

$R_{f}=R * k_{c}=5.1 \mathrm{k} * 47=239.7 \mathrm{k} \Omega \approx 240 \mathrm{k} \Omega$

\section{Circuito de Potencia}

El circuito de fuerza se muestra en la figura 8 , se ha utilizado un transistor TIP120 el cual permite amplificar la señal que envía el controlador y aplicarle al electroimán.

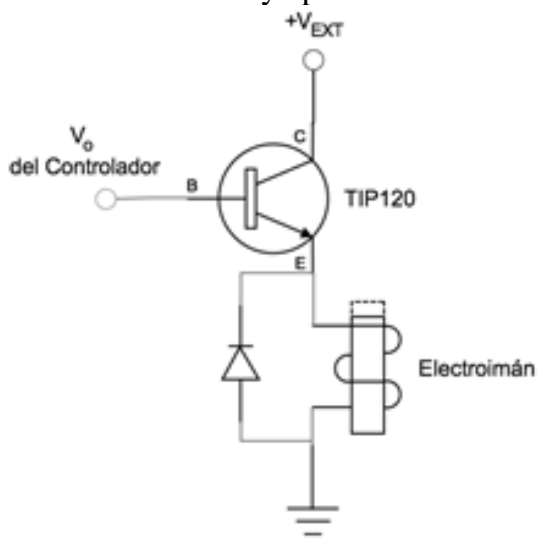

Fig. 8: Circuito de potencia del prototipo

\section{F. Tarjeta de Circuito Impreso}

Se diseñó en Eagle CAD una tarjeta de circuito impreso que consolida el circuito de la figura 6 y 8 que sirva como "shield" para la placa NUCLEO-L476RG, de tal forma que el prototipo sea sencillo de transportar. Este diseño e implementación se observa en la Fig. 9. Y 10 respectivamente

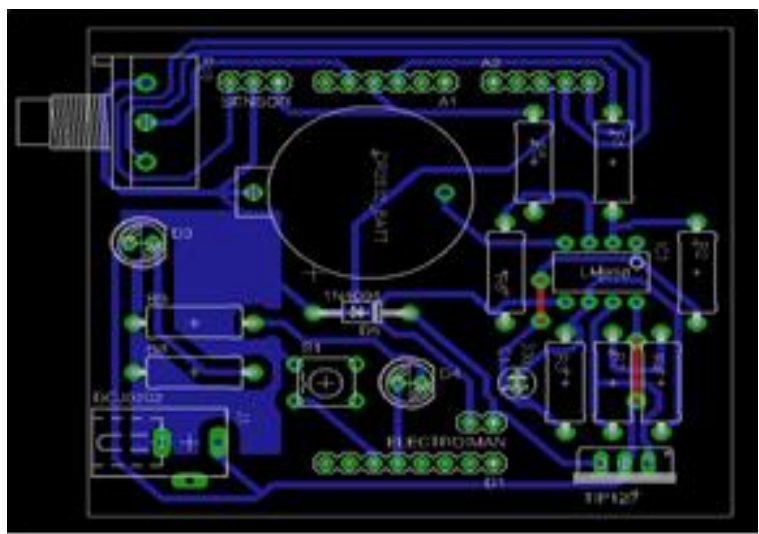

Fig. 9: Diseño de PCB del prototipo.

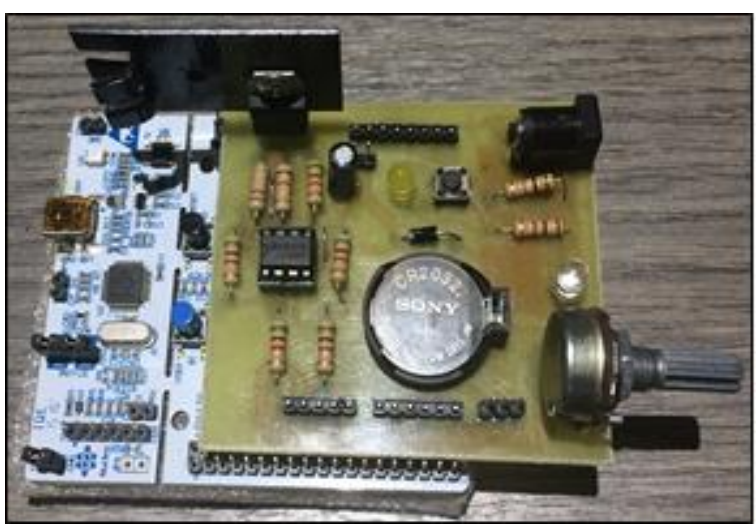

Fig. 10: PCB fabricada montada en la placa NUCLEO-L476RG.

$1^{\text {th }}$ LACCEI International Multi-Conference for Engineering, Education, and Technology: "Innovation in Education and Inclusion", 19-21 July 2018, Lima, Peru. 


\section{G. Estructura Física}

Finalmente, la estructura se construyó con MDF en base al diseño realizado en AutoCAD. El prototipo terminado junto a su estructura se observa en la Fig. 11.

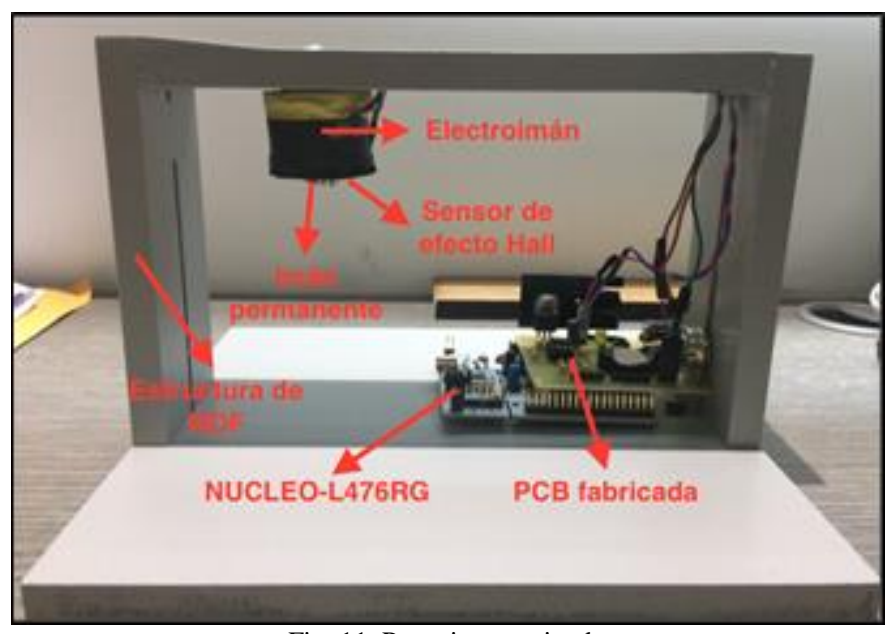

Fig: 11. Prototipo terminado

\section{H. Desarrollo de la Interfaz Gráfica}

La interfaz gráfica fue realizada con el editor de GUI de MATLAB, el cual ofrece un ambiente amigable programación muy parecido a Visual Basic, pero con la ventaja de contar con todas las funciones propias de MATLAB. En la Fig. 12 se puede ver el entorno de trabajo para el diseño del Layout de una GUI en este programa.

En la pantalla se observa el panel de Comunicación Serial, en donde se tiene los botones para Conectar, Desconectar, Actualizar, con la interfaz, además permite actualizar los puertos seriales, respectivamente. Desde el "pop up menú" de este panel se puede elegir el puerto serial en que se encuentra el dispositivo (NUCLEO-L476).

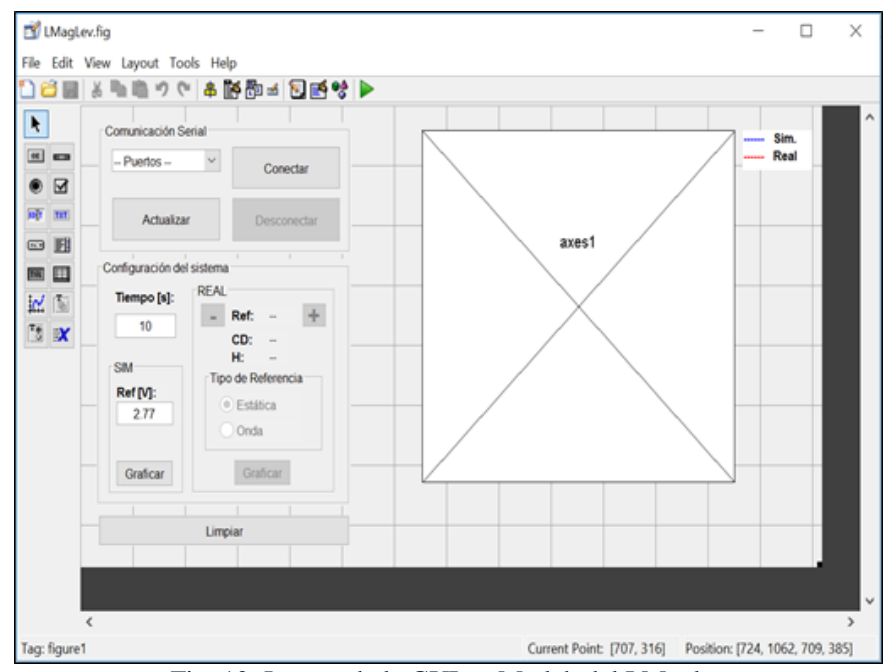

Fig. 12: Layout de la GUI en Matlab del LMaglev
En el panel Configuración del sistema, se tiene una casilla con la etiqueta Tiempo en donde se escribe el tiempo de simulación como también el tiempo de recolección de datos reales. En el subpanel SIM se tiene la casilla Ref en donde se podrá escribir la referencia que se quiere en voltios para la gráfica del modelo simulado. En el subpanel $R E A L$, se tiene en la parte superior dos botones etiquetados con $+\mathrm{y}-$, los cuales permiten aumentar o disminuir, respectivamente, el voltaje de referencia en el prototipo real. En este subpanel frente a la etiqueta $\operatorname{Ref}$ se podrá observar el valor de referencia en voltaje, frente a la etiqueta $C D$ se podrá observar el valor digital que se envía al DAC para llegar a esa referencia en voltios, y frente a la etiqueta $H$ se puede observar a la altura en que se encuentra el objeto levitando real. En la parte inferior del subpanel $R E A L$, se tiene otro subpanel Tipo de Referencia que consta de dos "radio buttons" que permiten al usuario seleccionar entre una referencia estática (el objeto se mantiene en un punto) y una referencia en onda (el objeto efectúa el movimiento de una onda cuadrada).

Finalmente, en el componente axes 1 se grafican las dos señales al pulsar cualquier botón Graficar: la simulada (representada con color azul) y la real (representada con color rojo). El botón Limpiar permite borrar las gráficas del axes1.

\section{RESULTADOS Y DISCUSIÓN}

En la figura 13 se aprecia el equipo funcionando, el disco de neodimio está levitando en $2 \mathrm{~cm}$ de distancia desde el electroimán hasta el objeto. En la figura 14 se observan la interface para el usuario, en esta gráfica, las dos señales de salida, la obtenida por medio de simulación y la que se obtiene de la planta real, se superponen, la diferencia entre las dos señales son las pequeñas fluctuaciones en la señal de la planta real, por efectos de ruido del sensor, pero no desestabilizan el sistema.

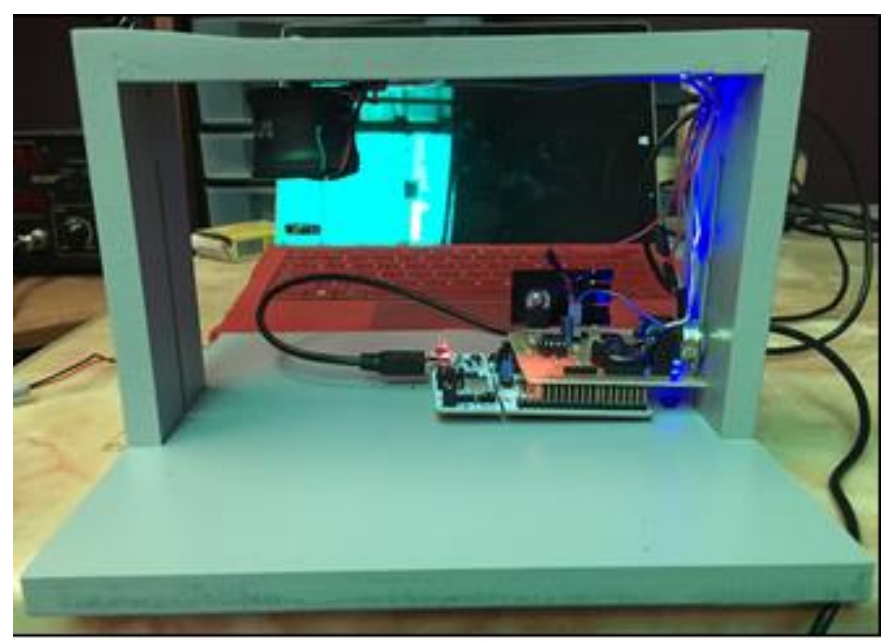

Fig. 13: Prototipo Funcionando

Las pruebas que se realizaron en tiempo real demostraron que el sistema real se aproxima bastante al sistema simulado,

$16^{\text {th }}$ LACCEI International Multi-Conference for Engineering, Education, and Technology: "Innovation in Education and Inclusion", 19-21 July 2018, Lima, Peru. 
con la diferencia que existe un cierto ruido, sin embargo, este ruido no desestabiliza el sistema. En la Fig. 13 se observa el prototipo funcionando.

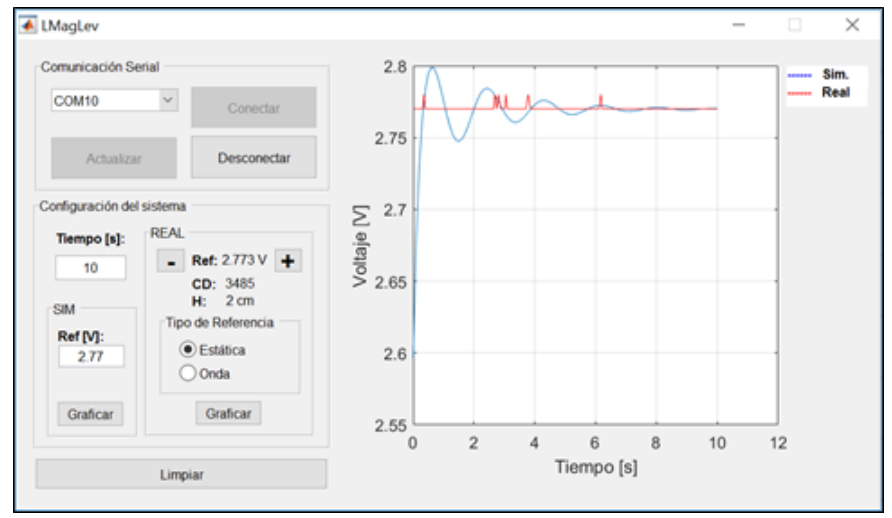

Fig. 14: Comparación de las repuestas del sistema real y la obtenida por simulación cuando el punto de operación es $2 \mathrm{~cm}(2.77$ V).

Cuando se realiza una prueba en un punto que es diferente al punto de linealización hay más fluctuaciones de la señal de salida, sin embargo, el controlador logra mantener el objeto levitando con pequeñas oscilaciones en la figura15 se observa estas pequeñas oscilaciones.

El trabajo de tesis de Guijarro y Vivar [23], muestran la construcción de un prototipo de levitador magnético, en el cual desarrollaron, además, una interfaz de usuario en LabView en donde se puede modificar las ganancias del controlador. Sin embargo, en este caso, un controlador PD no funcionó de manera apropiada como se ve en la Fig. 16.

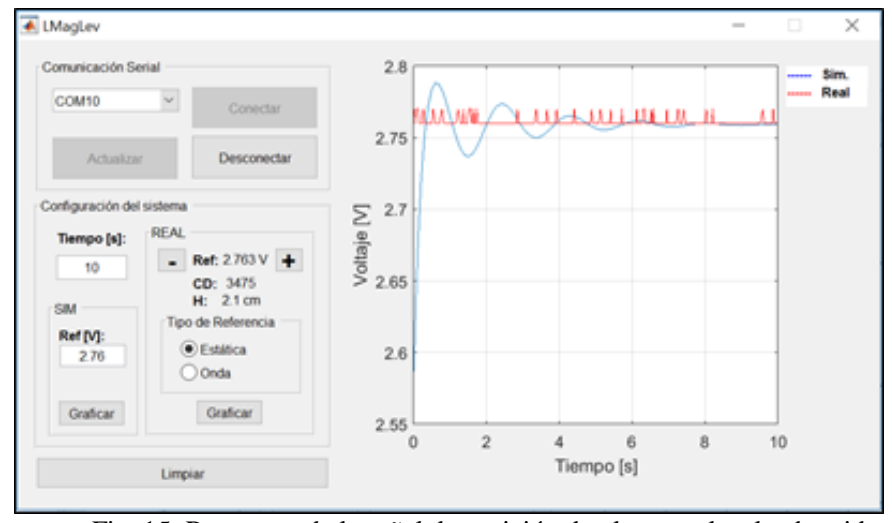

Fig. 15: Respuesta de la señal de posición de planta real vs la obtenida por simulación en un punto de operación de $2.1 \mathrm{~cm}(2.76 \mathrm{~V}-2.77 \mathrm{~V})$

Aunque el sistema mostrado en este artículo no cuenta con una interfaz gráfica en la que se pueda variar los parámetros del controlador, se puede variar el punto de operación para observar la estabilidad del sistema en puntos cercanos al punto de linealización. Por otro lado, el costo de fabricación planta de [23], es muy alto, en comparación a la presentada, lo cual la hace ideal para propósitos educativos.

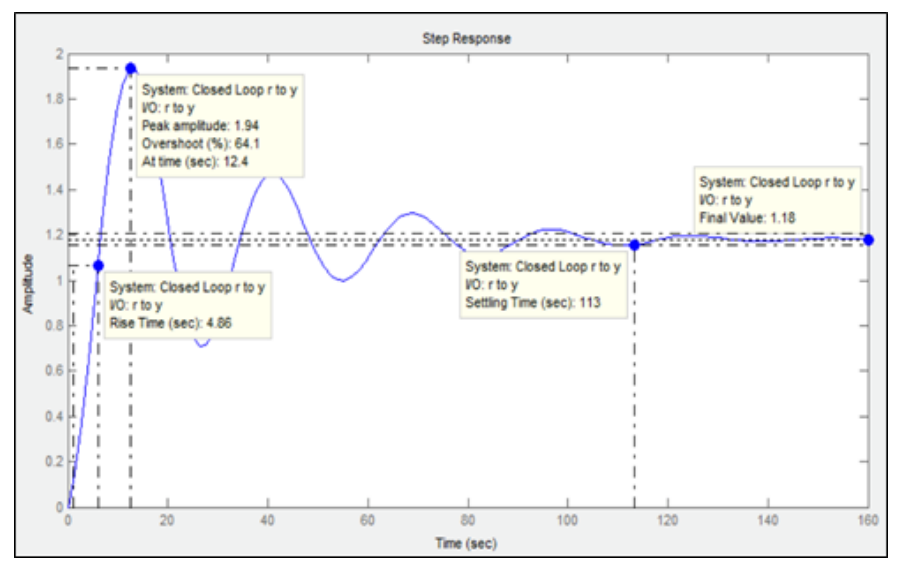

Fig. 16: Repuesta del sistema con controlador PD de los autores Guijarro y Vivar (2015)

\section{CONCLUSIONES}

En el presente trabajo, se logró diseñar e implementar un sistema de levitación magnético, que opera alrededor de un punto de operación de $2 \mathrm{~cm}$. A través del modelamiento correcto de la planta, es posible desarrollar y diseñar un controlador, el cual permita obtener una respuesta estable del sistema. El uso de la localización de raíces como método para el diseño del controlador es muy bueno, pues permite obtener una gama de diferentes controladores con distintas constantes, de entre los cuales se puede elegir aquella cuya incidencia en la planta provoque el resultado más cercano al deseado.

En algunos diseños, es suficiente y recomendable un controlador PD, pues un controlador PID podría provocar que la señal de mando del controlador supere los límites físicos de la planta, debido a la presencia de los errores mantenidos por la acción integral.

Cuando se tiene una planta cuyo comportamiento dinámico es no lineal, siempre se deberá tomar en cuenta que el valor alrededor del cual se linealiza la planta, será el que defina el rango de puntos de referencia posibles, así, para este sistema, el punto en el cual se linealizó fue $2 \mathrm{~cm}$ y $0.27 \mathrm{~A}$, por lo que los puntos de referencia no pueden estar muy alejados a esos $2 \mathrm{~cm}$. Se concluyó que para este prototipo desarrollado el punto de referencia sólo puede subirse o bajarse $10 \mathrm{~mm}$.

La interfaz de usuario creada en este trabajo facilita mucho el análisis de respuesta del sistema, pues de manera intuitiva el usuario puede observar la comparación de la respuesta del sistema real y simulado, como también cambiar la referencia en tiempo real.

Gracias a la sencillez y funcionalidad del prototipo presentado en este artículo, los costos de construcción del mismo son bastante bajos, así la reproducción de este sistema costaría aproximadamente 62 dólares, un precio aceptable y con buenas bondades que pueden ser explotadas en el ámbito educativo.

\section{REFERENCIAS}

[1] Berkelman, P., \& Dzadovsky, M. (2008). Magnet Levitition and trajectory following motion control using a planar array of cylindrical

$1^{\text {th }}$ LACCEI International Multi-Conference for Engineering, Education, and Technology: "Innovation in Education and 
coils. Recuperado el 2 de marzo de 2017, de www.me.hawaii.edu: http://www.me.hawaii.edu/HRIL/publications/magtraj-dscc08.pdf

[2] Abbadi, M. (2004). Modeling and Control of a Magnetic Levitation System. Recuperado el 2 de marzo de 2017, de cegt201.bradley.edu: http://cegt201.bradley.edu/projects/proj2003/maglev/docs/PDF/final.pdf

[3] INTECO. (2010). Magnetic Levitation System. Recuperado el 2 de marzo de 2017, de ee.sharif.edu: http://ee.sharif.edu/ linearcontrol89/Files/Lab/mls2em_um.pdf

[4] INTECO. (2017). Magnetic Levitation Systems. Recuperado el 20 de mayo de 2017, de www.inteco.com.pl: http://www.inteco.com.pl/products/magnetic-levitation-systems/

[5] Ahmad, I., \& Javaid, M. (2010). Nonlinear Model \& Controller Design for Magnetic Levitation System. Recuperado el 2 de marzo de 2017, de www.wseas.us: library/conferences/2010/Cambridge/ISPRA/ISPRA-53.pdf

[6] Becerril, R. (2003). Nonlinear Control Design for a Magnetic Levitation System. Recuperado el 2 de marzo de 2017, de www.control.utoronto.ca: http://www.control.utoronto.ca/theses/Rafael_Becerril_03.pdf

[7] Yu, W., \& Li, X. (2014). A Magnetic Levitation System for Advanced Control Education. Recuperado el 2 de marzo de 2017, de folk.ntnu.no: http://folk.ntnu.no/skoge/prost/proceedings/ifac2014/media/files/0147.pd

[8] Feedback Instruments. (2009). Magnetic Levitation Control Experiments. Recuperado el 2 de marzo de 2017, de www.cpdee.ufmg.br: http://www.cpdee.ufmg.br/ palhares/33-942s.pdf

[9] Zauba Technologies \& Data. (2013). Detailed Import Data of 33 lab equipment. Recuperado el 20 de mayo de 2017, de www.zauba.com: https://www.zauba.com/import-33+LAB+EQUIPMENT/hs-code-9023hs-code.html

[10] Zeltom. (2009). Electromagnetic Levitation System: Mathematical Model. Recuperado el 1 de marzo de 2017, de www.zeltom.com: http://zeltom.com/documents/emls_md.pdf

[11] Zeltom. (2017). Magnetic Levitation System. Recuperado el 25 de febrero de 2017, de zeltom.com: http://zeltom.com/products/magneticlevitation/emlscomponents

[12] Choudhary, S. (2014). Robust Feedback Control Analysis of Magnetic Levitation System. Recuperado el 2 de marzo de 2017, de www.wseas.org: http://www.wseas.org/multimedia/journals/systems/2014/a065702363.pdf

[13] Junaid, M., \& Ur, S. (2015). Observer Based Controller for Magnetic Levitation System. Recuperado el 2 de marzo de 2017, de ijcsse.org: http://ijcsse.org/published/volume4/issue2/p1-V4I2.pdf

[14] Milena, J. (2010). Control Lineal y No Lineal de un Levitador Magnético. Estudio Comparativo. Recuperado el 2 de marzo de 2017, de upcommons.upc.edu:

http://upcommons.upc.edu/bitstream/handle/2099.1/9923/Control\%20lin eal $\% 20$ y $\% 20$ no $\% 20$ lineal $\% 20 \mathrm{de} \% 20 \mathrm{un} \% 20$ levitador $\% 20$ magnetico.pdf? sequence $=1$

[15] Yu, W., \& Li, X. (2014). A Magnetic Levitation System for Advanced Control Education. Recuperado el 2 de marzo de 2017, de folk.ntnu.no: http://folk.ntnu.no/skoge/prost/proceedings/ifac2014/media/files/0147.pd $\mathrm{f}$

[16] OUZHUO. (2015). OH49E Series: Lineal Hall Effect IC. Recuperado el 25 de febrero de 2017, de www.zbling.com: http://www.zbling.com/datasheet/oh/OH49E-e.PDF

[17] Chico, M., \& Rondón, E. (2007). Diseño de un sistema de control para un levitador magnético que opere sumergido en fluidos con viscosidades y densidades sustancialmente diferentes a la del aire. Recuperado el 2 de marzo de 2017, de repositorio.uis.edu.co: http://repositorio.uis.edu.co/jspui/bitstream/123456789/3268/2/124098.p df

[18] Boundless Physics Boundless. (2016). Permanent Magnets. Recuperado el 2 de marzo de 2017, de www.boundless.com: https://www.boundless.com/physics/textbooks/boundless-physicstextbook/magnetism-21/magnetism-and-magnetic-fields-155/permanentmagnets-548-6079/
[19] STMicroelectronics. (2017). NUCLEO-L476RG. Recuperado el 25 de febrero de 2017, de www.st.com: http://www.st.com/en/evaluationtools/nucleo-1476rg.html

[20] STMicroelectronics. (2017). STM32L476RG. Recuperado el 25 de febrero de 2017, de www.st.com: http://www.st.com/en/microcontrollers/stm321476rg.html

[21] Carter, B., \& Brown, T. (2001). Handbook of Operational Amplifier Applications. Recuperado el 2 de marzo de 2017, de focus.ti.com: https://focus.ti.com/lit/an/sboa092a/sboa092a.pdf

[22] Yoon, M.-G., \& Moon, J.-H. (2016). A Simple Analog Controller for a Magnetic Levitation Kit. Recuperado el 1 de marzo de 2017, de www.ijert.org: http://www.ijert.org/view-pdf/14895/a-simple-analogcontroller-for-a-magnetic-levitation-kit

[23] Guijarro, J., \& Vivar, J. (2015). Diseño e implementación de un levitador electromagnético basado en un control PID utilizando LABVIEW. Recuperado el 2 de marzo de 2017, de dspace.ups.edu.ec: http://dspace.ups.edu.ec/bitstream/123456789/10425/1/UPS GT001498.pdf

16 $^{\text {th }}$ LACCEI International Multi-Conference for Engineering, Education, and Technology: "Innovation in Education and Inclusion", 19-21 July 2018, Lima, Peru. 